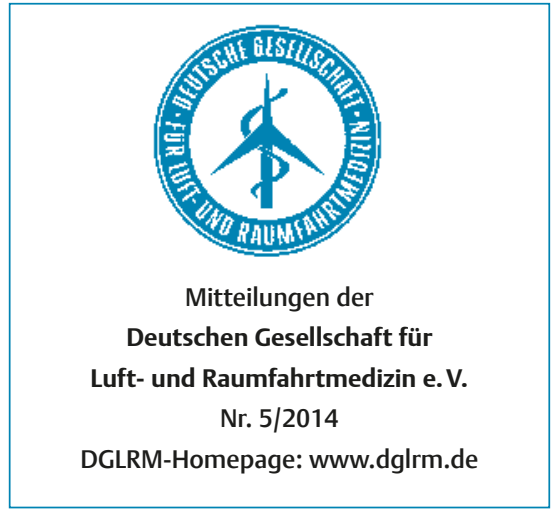

Geschäftsstelle der DGLRM

Christine Gens

Postfach 870204

13162 Berlin

E-Mail: christine.gens@dglrm.de Tel.: 0176/5624-2876

\section{Wir begrüßen als neue Mitglieder}

Dr. Walter Michael Klaus, Rastede Dr. Alexandros Livadas, Ruhpolding

\title{
Sehr geehrte Damen und Herren, liebe DG
}

diejenigen unter Ihnen, die die Gelegenheit hatten, die $4^{\text {th }}$ European Conference in Aerospace Medicine (ECAM 4), die die European Society of Aerospace Medicine (ESAM) vom 5. bis 7. September 2014 in Bukarest ausgerichtet hat, zu besuchen, sind sicher immer noch sehr beeindruckt von dem was dort geboten wurde. Die Organisatoren, allen voran Cristian Panait von der zivilen rumänischen Luftfahrtbehörde, haben keine Arbeit und Mühe gescheut, um diese Veranstaltung $\mathrm{zu}$ einem wahren Höhepunkt für Flugmediziner aus aller Welt werden zu lassen. Dies betraf das wissenschaftliche Programm ebenso wie auch das Begleitprogramm.

Unter dem Thema: „How we screen for and prevent health problems in aviation" sind Vortragssitzungen und Workshops abgehalten worden, die sich mit den Herausforderungen der Gesunderhaltung des Personals im Luftfahrtsektor und mit Möglichkeiten des Screenings von flugmedizinisch relevanten kognitiven Leistungsdefiziten bei Piloten beschäftigt haben.

Vorträge von Tony Evans, dem Direktor der flugmedizinischen Sektion der ICAO, Melchor Antunano, dem Direktor des Civil Aerospace Medical Institute
FAA, Philip J. Scarpa, dem amtierenden Präsidenten der AsMA, und Gary Kay, einem amerikanischen Neuropsychologen, aber auch von Rupert Gerzer, dem Direktor des Instituts für Luft- und Raumfahrtmedizin des DLR in Köln, und natürlich auch von Kevin Herbert, dem Präsidenten der ESAM, haben die Zuhörer regelrecht in ihren Bann gezogen.

Den Abschluss der Konferenz bildete am Sonntag, dem 7. September 2014, die Generalversammlung der ESAM auf der die turnusgemäßen Wahlen zum neuen Vorstand (EC - Executive Committee) stattfanden. Hier gab es ein paar personelle Veränderungen, jedoch ist es unserer Gesellschaft nun zum wiederholten Male gelungen, ihren Kandidaten im Vorstand zu platzieren. In der kommenden Wahlperiode wird Herr PD Dr. Jochen Hinkelbein das Amt des Schatzmeisters, das die DGLRM seit 2010 in der ESAM besetzt hat, weiterführen. Wir gratulieren ihm zu seiner Wahl und wünschen ihm viel Kraft bei der Ausübung dieses Amtes.

Nachdem sich die Beziehungen zwischen der ESAM und der AsMA in den letzten Jahren sehr positiv entwickelt haben und der ehemalige Präsident der

Blick ins Auditorium der ECAM 4.

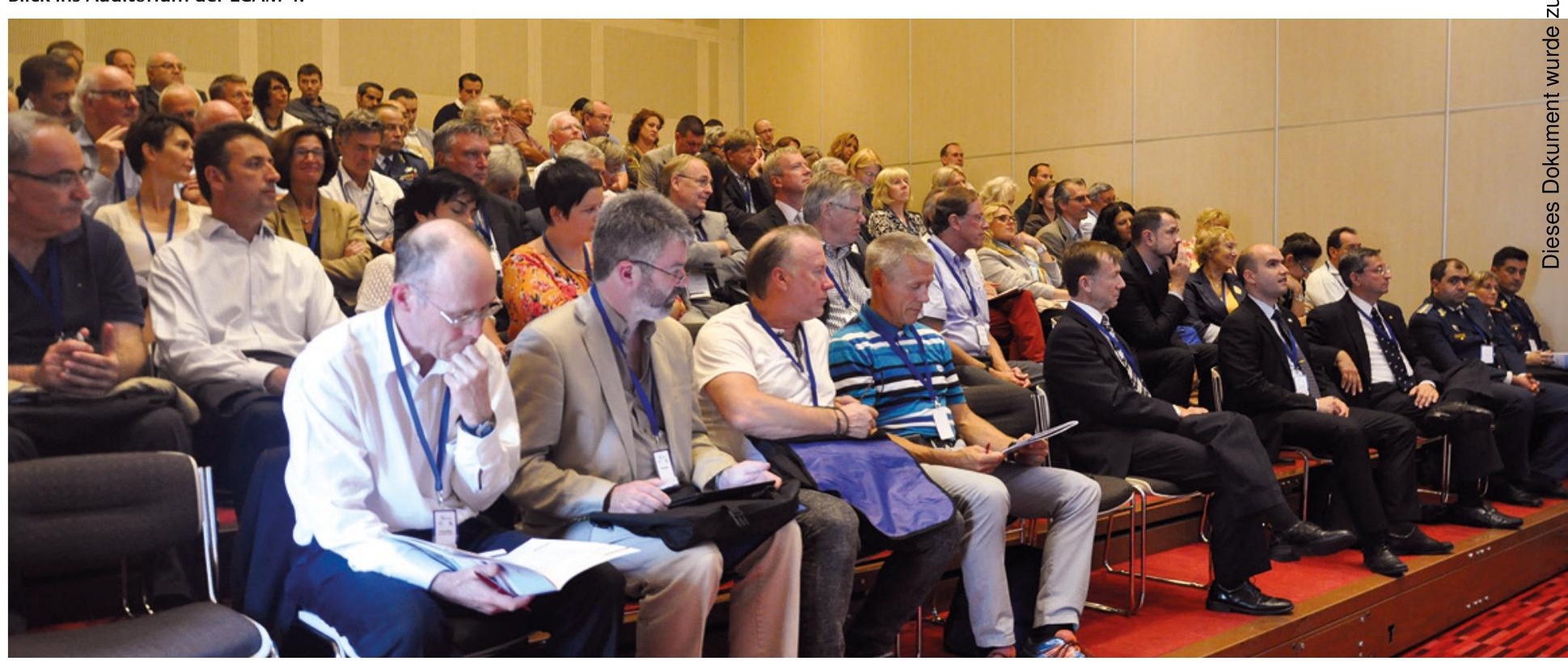




\section{LRM-Mitglieder,}

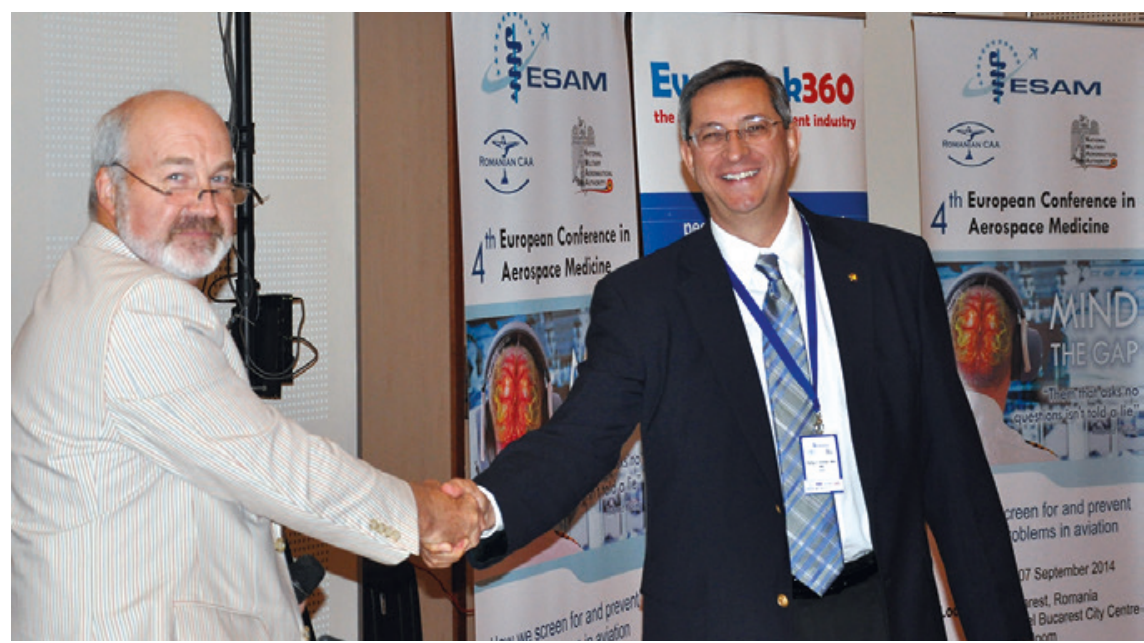

Dr. Kevin Herbert, Präsident der ESAM, und Dr. Philip Scarpa, Präsident der AsMA, beschließen eine gemeinsame Konferenz im Jahr 2016 anlässlich des 10-jährigen Bestehens der ESAM.

ESAM, Roland Vermeiren, inzwischen zum Vizepräsidenten der AsMA gewählt wurde, plant die ESAM ihre $5^{\text {th }}$ European Conference in Aerospace Medicine im Jahr 2016, bei der das 10-jährige Bestehen der ESAM begangen werden soll, zusammen mit dem ersten regionalen Meeting der AsMA in Europa auszurichten. Die nationalen Gesellschaften sind nun aufgerufen worden, sich um die Ausrichtung dieser Tagung zu bewerben. Ein erstes Angebot für Oslo als Tagungsort liegt von der norwegischen Gesellschaft bereits vor. Auch wir überlegen im Vorstand gerade, ob wir uns um die Ausrichtung bemühen sollten.

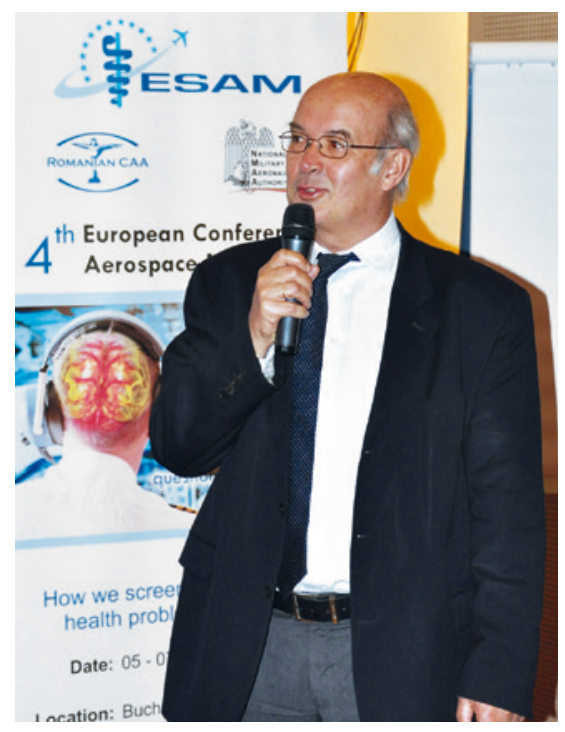

Professor Rupert Gerzer (DLR) bei seinem Vortrag.
Wissenschaftspreis 2014

In diesem Jahr gab es nach einer Phase der Stagnation im Jahr 2013 auf die Ausschreibung des nach Albrecht Ludwig Berblinger benannten Wissenschaftspreises der Deutschen Akademie für Flug- und Reisemedizin $\mathrm{gGmbH}$ erfreulich viele, vor allem aber auch fachlich sehr anspruchsvolle Bewerbungen, sodass die Auswahl des Siegers / der Siegerin für die Jury nicht leicht war. Der oder die Preisträgerin ist von einer Jury bereits ausgewählt worden. Der Preis wird am Nachmittag des zweiten Sitzungstags unserer diesjährigen DGLRM-Tagung in Heidelberg verliehen werden. Bis dahin wird der Name des Preisträgers/der Preisträgerin jedoch noch ein Geheimnis bleiben.

Auch im nächsten Jahr ist bereits wieder ein Wissenschaftspreis der Deutschen Akademie für Flug- und Reisemedizin gGmbH zur Förderung der Forschung im Bereich Luft- und Raumfahrtmedizin und verwandter Forschungsgebiete für hervorragende wissenschaftliche Arbeiten ausgeschrieben. Wir rufen daher vor allem junge Wissenschaftler auf, sich um diesen zu bewerben.

DGLRM-Jahrestagung in Heidelberg Für uns als Vorstand hat nun die heiße Phase der Vorbereitung unserer eigenen Jahrestagung begonnen. Das Programm verspricht interessant und anspruchsvoll sowie auch abwechslungsreich zu werden und wir hoffen, für jeden Geschmack etwas gefunden zu haben. Es wird Anfang Okto-
Ausschreibung Wissenschaftspreis

\section{Albrecht-Ludwig- Berblinger-Preis 2015}

Die Deutsche Akademie für Flug- und Reisemedizin gGmbH stiftet einen Preis zur Förderung der Forschung im Bereich Luft- und Raumfahrtmedizin, Reisemedizin und verwandte Forschungsgebiete.

Dieser Wissenschaftspreis wird jährlich für hervorragende wissenschaftliche Arbeiten verliehen und richtet sich besonders an den wissenschaftlichen Nachwuchs.

Der Preis besteht aus einer Urkunde und einer Prämie in Höhe von 10000 Euro.

Bewerbungen für den Wissenschaftspreis im Jahr 2015 müssen bis zum 28. Februar 2015 dem Wissenschaftlichen Beirat der Deutschen Akademie für Flug- und Reisemedizin gGmbH, Lufthansabasis FRA PM/F, 60546 Frankfurt, zugesandt werden.

Der Bewerber sollte darlegen,

- warum er sich um den Preis bewirbt,

- die Arbeit in 3-facher gebundener Ausfertigung mit Lebenslauf einreichen,

- nur eine Arbeit/Publikation pro Ausschreibung einreichen,

- die Arbeit bisher nicht bei einer anderen Institution etc. zur Prämierung vorgelegt haben.

ber auf unserer Homepage abrufbar sein. Wir werden einen Teil der Vorträge in Englisch anbieten, damit die Vertreter des Vorstands der European Society of Aerospace Medicine (ESAM), die unser Programm mit einer eigenständigen Session bereichern werden, auch einen eigenen ideellen Gewinn aus der Tagung ziehen können. Ich danke deshalb allen Kolleginnen und Kollegen, die sich bereit erklärt haben, ihren Vortrag in englischer Sprache zu halten. Aufgrund unserer nochmaligen Werbeaktion haben wir nun auch genügend Poster- 


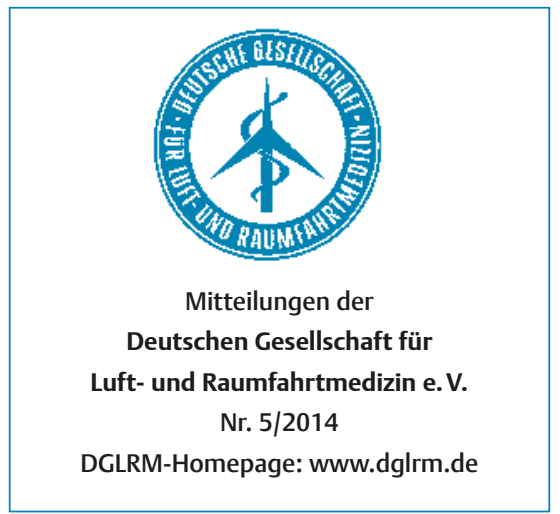

\section{Kongresse 2014}

52. Jahrestagung der Deutschen Gesellschaft für Luft- und Raumfahrtmedizin (DGLRM)

Termin: 24.10.-25.10.2014

Ort: Heidelberg

Internet: www.dglrm.de

\section{Verantwortlich für den Inhalt} der DGLRM-Seiten

Deutsche Gesellschaft für Luft- und Raumfahrtmedizin e. V. (DGLRM) OFA PD Dr. Carla Ledderhos, Präsidentin

Zentrum für Luft- und Raumfahrtmedizin der Luftwaffe

Str. der Luftwaffe 322

82242 Fürstenfeldbruck

carlaledderhos@bundeswehr.org

Redaktionsschluss: 30.09.2014

Bankverbindung

Deutsche Bank Hamburg

Konto-Nr.: 388948200

BLZ: 20070000

IBAN: DE 63200700240388948200

BIC: DEUTDEDBHAM

Änderungen für die Mitgliederkartei bitte an: OL Christine Gammel

Zentrum für Luft- und Raumfahrtmedizin

der Luftwaffe

Str. der Luftwaffe 322

82242 Fürstenfeldbruck

christinegammel@bundeswehr.org anmeldungen für den Kongress zusammen, um wieder einen Posterpreis vergeben zu können. Wir planen in diesem Jahr, den 3 Preisträgern die Möglichkeit zu geben, ihr Poster in einem Kurzvortrag von 5 bis 10 Minuten dem Publikum nahezubringen.

Auch Fortbildungsstunden und -punkte vom LBA und der LÄK Baden-Württemberg werden wieder vergeben werden können. Nicht zuletzt bleibt noch daran zu erinnern, dass unsere alljährliche Mitgliederversammlung am Freitag, den 24. Oktober 2014, in der Print Media Academy stattfinden wird und ich Sie hiermit herzlich dazu einladen möchte.

Anmeldungen zur Tagung sind über unser Anmeldeformular, das Sie auf unserer
Homepage www.dglrm.de abrufen können, möglich.

In der Hoffnung so viele Mitglieder wie möglich auf unserer diesjährigen Jahrestagung in Heidelberg begrüßen zu dürfen, verbleibe ich mit den besten Wünschen sowie mit herzlichen Grüßen aus Fürstenfeldbruck

Ihre

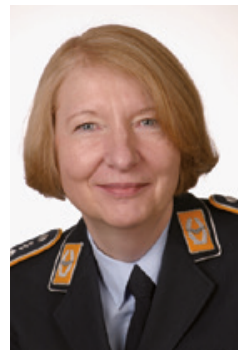

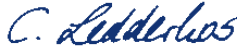

Carla Ledderhos, Fürstenfeldbruck

\section{Lehrgänge 2014}

DAF-Refresher-Seminar Flugmedizin

Dt. Akademie für Flug- und Reisemedizin

Termin: 31.10.-02.11.2014

Ort: Seeheim-Jugenheim, Lufthansa Training \& Conference Center

Kontakt: Sigrid Froese, Tel.: +49 (0)6969691222

Internet: www.flugmed.org

Flugmedizinischer Lehrgang 1

Basic (englisch)

Zentrum für Luft- und Raumfahrt-

medizin der Luftwaffe

Termin: 10.11.-19.11.2014

Ort: $\quad$ Fürstenfeldbruck

Kontakt: Generalarzt der Luftwaffe ZentrLuRMedLw Dez. III 2 a

Flughafenstr. 1

51147 Köln

Tel.: +49 (0)22039081697
Flugmedizinischer Lehrgang 1

Advanced (englisch)

Zentrum für Luft- und Raumfahrt-

medizin der Luftwaffe

Termin: 20.11.-02.12.2014

Ort: $\quad$ Fürstenfeldbruck

Kontakt: Generalarzt der Luftwaffe ZentrLuRMedLw Dez. III 2 a Flughafenstr. 1 51147 Köln Tel.: +49 (0)22039081697

EUSAM - Advanced course 24 (englisch)

European School of Aviation Medicine

Termin: 29.11.-07.12.2014

Ort: $\quad$ Medizinischer Dienst der Lufthansa auf der Lufthansa-Basis, Flughafen Frankfurt

Kontakt: Sigrid Froese, Tel: +49 (0)69 69691222 Internet: www.flugmed.org / www.eusam.org 